\title{
Development of Virtual Surveys for the COVID-19 Wave of the AGELESS Longitudinal Study in Malaysia
}

\author{
Kiirtaara Aravindhan ${ }^{\text {a }}$ Sumaiyah Mat ${ }^{a, c}$ Tengku Aizan Hamid ${ }^{b}$ \\ Suzana Shaharc Abu Bakar Abdul Majeed ${ }^{d}$ Pei-Lee Teh ${ }^{\mathrm{e}, \mathrm{f}}$ \\ Kalavathy Ramasamy ${ }^{g}$ Devinder Kaur Ajit Singh ${ }^{c}$ Maw Pin Tan ${ }^{a, h, i}$
}

\begin{abstract}
${ }^{a}$ Ageing and Age-Associated Disorders Research Group, Faculty of Medicine, University of Malaya, Kuala Lumpur, Malaysia; ${ }^{b}$ Malaysian Research Institute on Ageing, Universiti Putra Malaysia, Serdang, Malaysia; ' ${ }^{C}$ entre for Healthy Aging and Wellness, Faculty of Health Sciences, Universiti Kebangsaan Malaysia, Kuala Lumpur, Malaysia; ${ }^{\text {Brain }}$ Degeneration and Therapeutics Group, Faculty of Pharmacy, Universiti Teknologi MARA (UiTM), Bandar Puncak Alam, Malaysia; ${ }^{\mathrm{e} G e r o n t e c h n o l o g y ~ L a b o r a t o r y, ~ M o n a s h ~ U n i v e r s i t y ~ M a l a y s i a, ~ B a n d a r ~ S u n w a y, ~ M a l a y s i a ; ~ f S c h o o l ~ o f ~}$ Business, Monash University Malaysia, Bandar Sunway, Malaysia; ${ }^{9}$ Collaborative Drug Discovery Research (CDDR) Group, Faculty of Pharmacy, Universiti Teknologi MARA (UiTM), Puncak Alam, Malaysia; ' $D i v i s i o n$ of Geriatric Medicine, Department of Medicine, Faculty of Medicine, University of Malaya, Kuala Lumpur, Malaysia; 'Department of Medical Sciences, School of Healthcare and Medical Sciences, Sunway University, Bandar Sunway, Malaysia
\end{abstract}

\section{Keywords}

Coronavirus disease 2019 - Virtual survey · Older people · Technology · Frailty

\footnotetext{
Abstract

Background: Rapid population aging occurring in developing nations necessitates innovation to ensure we continue to gain ground on aging research despite pandemic threats. While developed nations have resorted to virtual communications, this is challenging in developing nations due to poor internet connectivity and digital literacy. Objectives: The aim of this study was to determine the feasibility of virtual data collection for a longitudinal study of aging assessing cognitive frailty in a middle-income Southeast Asian country. Methods: The Transforming Cognitive Frailty into LaterLife Self-Sufficiency (AGELESS) longitudinal study of aging involved community-dwelling participants aged 60 years and above. A semi-structured focus group discussion was
}

conducted via videoconferencing with selected representatives from existing participants. The survey instrument was compiled during a hybrid meeting and refined using a virtual Delphi process involving 51 AGELESS investigators. The final draft survey and recruitment strategy were then piloted among selected participants. Results: Twelve individuals participated in the virtual focus group interview. Smartphone, tablet computer, laptops, and desktop personal computers were used for information gathering, communication, banking, shopping, leisure, religion, and education, within this group. The survey instrument was redacted from 362 items in 18 sections to 141 items in 12 sections through 3 virtual Delphi rounds facilitated by email, social media messaging, and videoconferencing which attracted 213 comments. Of 45 participants selected for the pilot survey, 30 were successfully contacted after one attempt and 18 completed the survey. Cognitive frailty was present in $13 \%$, cognitive impairment in $20 \%$, frailty in $20 \%$, and $47 \%$ were robust. Conclusion: A virtual survey instrument was devel-
Correspondence to:

Maw Pin Tan, mptan@ummc.edu.my 
oped for the AGELESS longitudinal survey of aging which was vital for determining the effects of the COVID-19 pandemic on our older population as well as sustaining research into aging despite barriers posed by the pandemic.

(C) 2021 S. Karger AG, Basel

\section{Introduction}

The unprecedented arrival of the coronavirus disease 2019 (COVID-19) pandemic led to the imposition of lockdowns in many countries globally. Unfortunately, research not related to treatment and prevention of COVID-19 was arguably less prioritized than economic and educational activities, in terms of standard operating procedures for physical distancing and infection control. This inevitably encumbered all current and prospective research plans within research communities globally [1], including that of the Transforming Cognitive Frailty into Later-Life Self-Sufficiency (AGELESS) longitudinal study on aging in Malaysia.

Nonetheless, issues pertaining to the rapidly aging population, in Malaysia, remain impervious to the COVID-19 pandemic, with relentless increase in the population aged 65 years and over from $5 \%$ in 2010 to $14.5 \%$ by 2040 [2]. Innovations are much required to ensure aging research persists. Smartphone penetration rate exceeding $90 \%$ among the Malaysian population presents the possibility of embracing virtual communication methods for our clinical study [3]. Nevertheless, concerns surrounding older adults' ability to use information technology remain [4]. Therefore, the aim of this study was to determine the feasibility of virtual data collection for a longitudinal study of aging in a middle-income country in Southeast Asia.

\section{Materials and Methods}

Two Malaysian longitudinal studies of aging involving community-dwelling older adults aged above 55 years recruited in 2013-2015 were merged to form AGELESS $[5,6]$. Participant contact details collected during the initial wave were readily available. Development and evaluation of the AGELESS virtual survey involved public engagement through a focus group interview, modified Delphi process, and pilot survey.

\section{Focus Group Interview}

A participant representative from the original cohort was contacted, with additional participants recruited through purposive sampling and snowballing based on familiarity with technology and likelihood of participation in group discussions. A semi-structured interview was then conducted through a videoconferencing platform (Google Meet ${ }^{\mathrm{TM}}$, Google Inc., USA). The session was recorded with permission, transcribed, and a thematic analysis conducted.

\section{Modified Delphi}

A 1-day workshop was conducted before lockdown during which a list of potential questions on sociodemographic, cognition, frailty, psychology, mobility, medical history, medications, health utilization, and caregiving was collated. A modified Delphi process was conducted through electronic mail, social media messaging, and videoconferencing. Investigators rated each question according to importance and appropriateness for virtual interviews and recorded free-text comments. Amendments were made according to the document which was recirculated until consensus was achieved.

\section{Pilot Survey}

Participants with telephone numbers were selected randomly from our existing cohort. The pilot instrument was administered by trained interviewers via telephone or video call. Only 1 attempt was made to contact participants, and only individuals fluent in the English language were included. Responses were recorded electronically. Feedback relevant to acceptability of virtual survey and survey measures was obtained from enumerators and participants. Cognitive frailty, which is the primary outcome of the cohort study, was defined as the presence of cognitive impairment and frailty. Cognition was assessed using the Montreal Cognitive Assessment for the Blind (MoCA-Blind) [7]. The FRAIL scale was utilized with frail defined as the presence of 3 or more of the components of fatigue, ambulation, resistance, illness, and weight loss [8].

\section{Results}

\section{Focus Group Interview}

Twelve participants, who utilized information technology for information gathering, communication, banking, shopping, leisure, religion, and education using smartphones, tablet computers, laptops, and personal computers, attended the virtual focus group interview. One participant mentioned participating in online group prayers: "With my prayer group, we do a lot of Zoom-ing" (69-year-old housewife).

Several individuals participated in online banking and shopping:"E-Payment. I use Touch N Go e-wallet. That's very convenient" (64-year-old retired dentist).

Some found it useful for learning:"I will Google and look into various nurseries and the type of plants. It's a real zig-zag way of learning" (70-year-old retired nurse).

Participants expressed reservations toward video interviews and some preferred self-administered online questionnaires. There were concerns that other older adults may be not as familiar with the use of technology. 


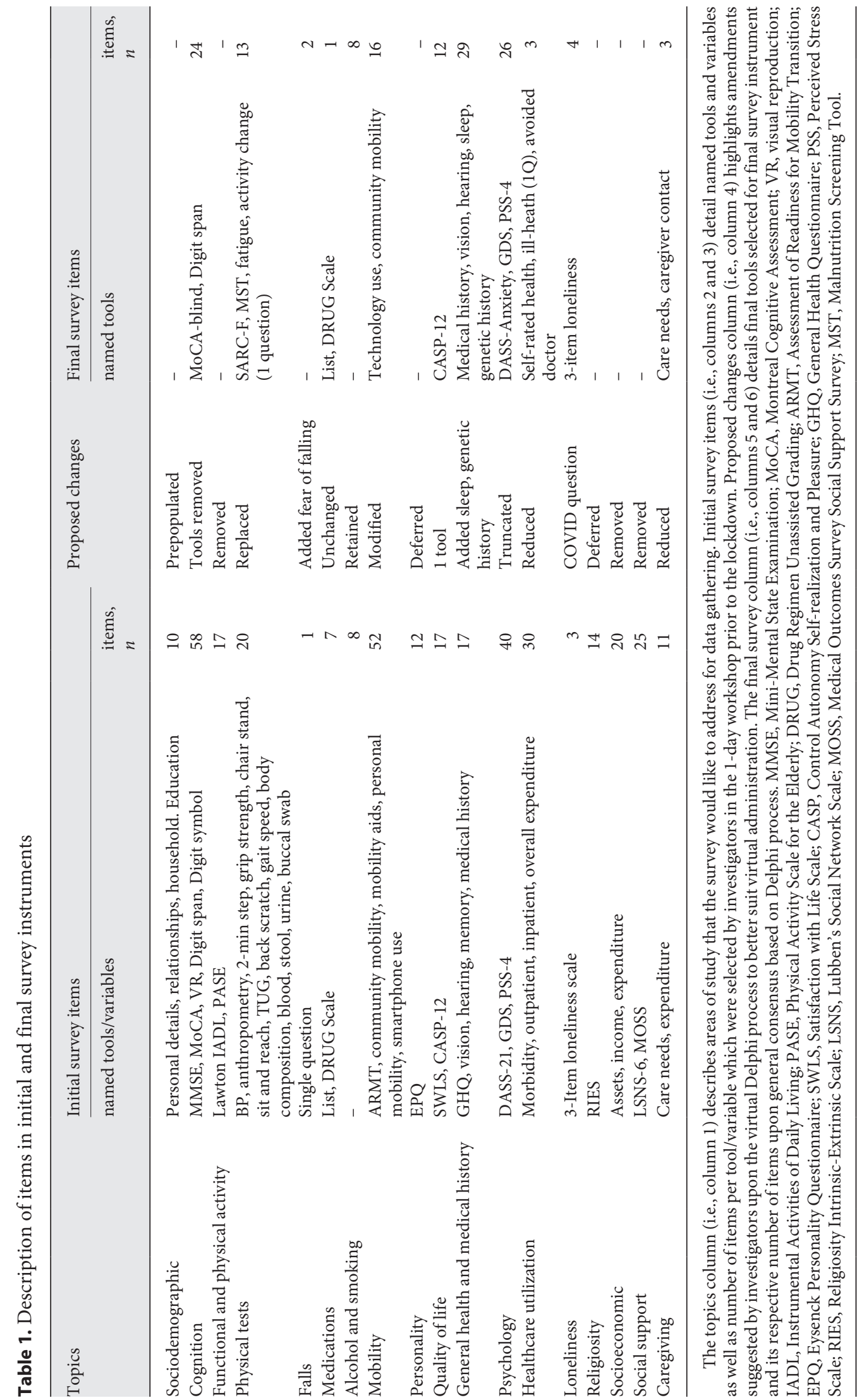




\section{Survey Instrument}

The initial 362 questions within 18 topic sections underwent 3 modified Delphi rounds, drawing 213 comments. Items on psychological wellbeing, sleep, quality of life, and loneliness among other pandemic-related items received higher ratings. Items involving physical assessments received lower ratings and were replaced or removed. Some items were removed or deferred to future waves based on previously available information, likelihood of change, and applicability of tools during the lockdown (e.g., outdoor activity). The emergent study instrument comprised 141 items within 12 topic sections. Table 1 lists the topics and tools that were initially selected and the final survey instrument.

\section{Pilot Survey}

Of 45 selected participants, 30 (67\%) were successfully contacted. Twelve (40\%) of the 30 respondents were either unavailable, declined, or excluded for language difficulties. Of the 18 individuals interviewed, $83 \%$ owned a smartphone, while $20 \%$ had prior experience with videoconferencing. Query on the interview delivery method found $67 \%$ preferred telephone interviews, $22 \%$ video calls, and $11 \%$ self-administered online surveys. Most participants found the 80-min interview too lengthy, while functional and physical activity questions were considered irrelevant. Results were analyzed for the primary outcome, cognitive frailty, among 15 participants, with 3 excluded due to missing data. Cognitive frailty was present in 2 (13\%), frailty only in $3(20 \%)$, cognitive impairment only in $3(20 \%)$, and 7 (47\%) were robust.

\section{Discussion}

Research in older adults had only been conducted face-to-face before pandemic in our setting, and assistance with self-administered questionnaires was often required with lower literacy and higher dependency levels [9]. But, this feasibility study on virtual interviews in older Malaysians conducted at an accelerated pace over a 2-month period yielded successfully completed interviews for the AGELESS longitudinal study of aging, with adequate data for classification into cognitive frailty categories.

The focus group exercise was useful in determining our older adults' ability to use digital communications and openness toward virtual interviews. Steady rise in tech-savviness among older adults has been observed prior to the pandemic, but a leap in digital literacy oc- curred as a result of lockdown measures due to necessity [10]. As such, most of our focus group participants owned $>1$ digital communication device. These findings provide a fresh perspective on the use of technology among older adults, enabling effective implementation of Internet of Things-enabled technologies within the population [11].

Our focus group suggested using self-administrated questionnaires, but our pilot participants favored telephone interviews above videoconferencing and self-administration. Telephone interviews have the propensity to generate information-rich responses, even for sensitive subject matters [12]. Further, the use of virtual interviews also addresses safety and transportation issues that often complicate home-based interviews.

The modified virtual Delphi exercise proved to be an inexpensive and unbiased method for devising an inclusive questionnaire. Our questionnaire, however, needed to be concise to enhance response rate and data quality [13]. Participants wanted to complete the questionnaire in one sitting despite the length of the questionnaire. Therefore, it was important to achieve a balance between details and brevity. Single questions may be favored above assessment scales to reduce the likelihood of noncompletion. Nonetheless, researchers need to ensure reliable and valid measurement of the concepts in the study.

Low response rates were recorded for virtual interviews as only English language interviews were conducted within 1 week, and only 1 attempt was made to contact participants due to time and resource limitations. The actual interview will incorporate Malay, Chinese, and Tamil, commonly spoken local languages. Selection bias was unavoidable as those familiar with videoconferencing and modern telecommunications were more likely to be included. Appropriate adjustments during data analysis and interpretation are necessary to account for these potential differences.

\section{Conclusion}

Our study has confirmed the feasibility of virtual interviews as a means of reaching out to older research participants currently shielded due to their vulnerability to COVID-19, in addition to lockdown restrictions. Wave 1 interviews for AGELESS will now proceed with the valuable information provided by this exercise to ensure that research in older adults continues despite the COVID-19 pandemic and to measure the effects of the lockdown measures on our older population. 


\section{Acknowledgments}

We are grateful for the contributions of all AGELESS investigators in aiding the Delphi process and data collection: Irraivan Elamvazhuti, Nor I'zzati Saedon, Kejal A/P Hasmukharay, Azrina Binti Abd Aziz, Norlisah Binti Mohd Ramli, Kartini Rahmat, Mazlyfarina Mohamad, Normala Mesbah, Azlina Bt Ahmad Annuar, Hazlina Mahadzir, Fakhrul Zaman Rokhani, Nor Fadilah Rajab, Lim Weng Marc, Lila Iznita Binti Izhar, Chengkai Lu, Shahrul Bahyah Kamaruzzaman, Rahimah Ibrahim, Siti Anom Ahmad, Sharifah Azizah Haron, Amrizal Muhammad Nur, Aidalina Mahmud, Roshanim Koris, Asmidawati Ashar, Nur Syazwani Mazlan, Hanis Mastura Yahya, Khor Hui Min, Lim Siong Meng, Nasreen Badruddin, Neoh Chin Fen, Ng Min Hwei, Rajesh Ramasamy, Tan Ai Huey, Thamil Selvee Ramasamy, Chin Aivyrn, Shahrul Azman Mohd Noah, Norhayati Ibrahim, Nazlena Mohamad Ali, Ponnusamy Subramaniam, Arimi Fitri Mat Ludin, Divya Vanoh, Shaun Lee Wen Huey, V Santha A. Vaithilingam, Tan Kit Mun, Zainora Mohammed, Asmidawati Ashari, Lim Weng Marc, Wan Syafira Ishak, Chong Chun Yong, Deepa Alex, and Jennifer Oxley.

\section{Statement of Ethics}

Ethical approval was obtained from the University of Malaya Medical Research Ethics Committee, prior to commencement of the study (MREC ID: 201912231-8121). Informed consent to participate was not directly obtained but inferred by completion of the questionnaire/participation in the interview.

\section{Conflict of Interest Statement}

The authors have no conflicts of interest to declare.

\section{Funding Sources}

The Transforming Cognitive Frailty to Later-Life Self-sufficiency (AGELESS) study is funded by the Ministry of Higher Education Malaysia Long-Term Research Grant Scheme (LRGS/1/ 2019/UM//1/1)

\section{Author Contributions}

K.A., S.M., and T.M.P. were responsible in coordinating focus group interview, Delphi technique, and pilot study. T.A.H., S.S., A.B.A.M., K.R., T.P.L., and D.K.A.J. facilitated in recruitment of pilot participants and enumerators besides assisting in data collection.

\section{Data Availability Statement}

Meta-data that support the findings of this study will be publicly available once cohort description is published but are available from the corresponding author (T.M.P.) upon reasonable request.

\section{References}

1 Haleem A, Javaid M, Vaishya R, Deshmukh SG. Areas of academic research with the impact of COVID-19. Am J Emerg Med. 2020; 38(7):1524-6.

2 Department of Statistics Malaysia. Population projection (Revised), Malaysia, 20102040 [press release]. Department of Statistics Malaysia; 2016 Nov 4. Available from: https: // www.dosm.gov.my/v1/index. $\mathrm{php} ? \mathrm{r}=$ column/pdfPrev\& id $=\mathrm{Y} 3 \mathrm{kwU} 2 \mathrm{tSNVF}$ DOWp1YmtZYnhUeVBEdz09.

3 Muller J. Smartphone penetration rate as share of the population in Malaysia from 2015 to 2025. Statista; 2020 July. Available from: https: //www.statista.com/statistics/625418/ smartphone-user-penetration-in-malaysia.

4 Lobe B, Morgan D, Hoffman KA. Qualitative data collection in an era of social distancing. IntJQualMethods.2020;19:160940692093787.

5 Shahar S, Omar A, Vanoh D, Hamid TA, Mukari SZ, Din NC, et al. Approaches in methodology for population-based longitudinal study on neuroprotective model for healthy longevity (TUA) among Malaysian Older Adults. Aging Clin Exp Res. 2016 Dec;28(6): 1089-104.
6 Lim LM, McStea M, Chung WW, Nor Azmi N, Abdul Aziz SA, Alwi S, et al. Prevalence, risk factors and health outcomes associated with polypharmacy among urban community-dwelling older adults in multi-ethnic Malaysia. PLoS One. 2017 Mar 8;12(3):e0173466.

7 Nasreddine ZS, Phillips NA, Bédirian V, Charbonneau S, Whitehead V, Collin I, et al. The Montreal Cognitive Assessment, MoCA: a brief screening tool for mild cognitive impairment. J Am Geriatr Soc. 2005;53(4):695-9.

8 Morley JE, Malmstrom TK, Miller DK. A simple frailty questionnaire (FRAIL) predicts outcomes in middle aged African Americans. J Nutr Health Aging. 2012;16(7):601-8.

9 Hairi NN, Bulgiba A, Cumming RG, Naganathan V, Mudla I. Prevalence and correlates of physical disability and functional limitation among community dwelling older people in rural Malaysia, a middle income country. BMC Public Health. 2010;10(1):492.
10 Finn C. How tech is helping elderly fight coronavirus lockdown loneliness. Aljazeera.com 2020. Available from: https://www.aljazeera. com/news/2020/4/6/how-tech-is-helping-elderly-fight-coronavirus-lockdown-loneliness.

11 Singh RP, Javaid M, Haleem A, Suman R. Internet of things (IoT) applications to fight against COVID-19 pandemic. Diabetes \& Metabolic Syndrome. Clin Res Rev. 2020 Jul; 14(4):521-4.

12 Drabble L, Trocki KF, Salcedo B, Walker PC, Korcha RA. Conducting qualitative interviews by telephone: lessons learned from a study of alcohol use among sexual minority and heterosexual women. Qual Soc Work. 2016;15(1):118-33

13 Burgard T, Bošnjak M, Wedderhoff N. Response rates in online surveys with affective Disorder participants. Z Psychol. 2020; 228(1):14-24. 